\title{
Tumor Implant
}

National Cancer Institute

\section{Source}

National Cancer Institute. Tumor Implant. NCI Thesaurus. Code C151403.

A nodule of a malignant tumor that develops at a site in a body cavity at a distance from the initial site of cancer. This is a finding typical of ovarian cancer. 